\title{
Cardiovascular imaging in cardio-oncology
}

\author{
Amir Abbas Mahabadi ${ }^{1}$, Christoph Rischpler ${ }^{2}$ \\ ${ }^{1}$ Department of Cardiology and Vascular Medicine, West German Heart and Vascular Center, University Hospital Essen, ${ }^{2}$ Department of Nuclear \\ Medicine, University Hospital Essen, University of Duisburg-Essen, Essen, Germany \\ Contributions: (I) Conception and design: All authors; (II) Administrative support: All authors; (III) Provision of study materials: All authors; (IV) \\ Collection and assembly of data: All authors; (V) Data analysis and interpretation: All authors; (VI) Manuscript writing: All authors; (VII) Final \\ approval of the manuscript: All authors. \\ Correspondence to: Amir Abbas Mahabadi, MD. Hufelandstr. 55, 45147 Essen, Germany. Email: amir-abbas.mahabadi@uk-essen.de.
}

\begin{abstract}
Cancer therapy may lead to cardiovascular complications and can promote each aspect of cardiac disease manifestation, such as vascular disease including coronary heart disease, myocardial diseases including heart failure, structural heart diseases including valvular heart diseases, and rhythm disorders. All potential complications of cancer therapy onto the cardiovascular system require imaging for diagnostic workup as well as monitoring of therapy. Transthoracic echocardiography (TTE) is the most frequently used tool for assessment of cardiac function during or after cancer therapy in daily clinical routine. With modern techniques like strain analysis, echocardiography allows to detect a variety of cardiac diseases as caused by cancer therapy even at subclinical stages. For further workup, specific imaging techniques including nuclear imaging are needed in a multimodality imaging approach to in detail characterize the underlying pathophysiology and to improve the management of the patients. Therefore, the field of imaging in cardiooncology is rapidly growing. This review article will give an overview about existing literature regarding the role of imaging in the diagnostic evaluation and management of therapy in patient with prior or ongoing cancer therapy.
\end{abstract}

Keywords: Cardiovascular imaging; cardio-oncology; echocardiography; computed tomography (CT); magnetic resonance imaging (MRI); nuclear cardiac imaging; multimodality imaging

Submitted Oct 12, 2018. Accepted for publication Oct 25, 2018.

doi: $10.21037 /$ jtd.2018.10.92

View this article at: http://dx.doi.org/10.21037/jtd.2018.10.92

\section{Introduction}

Cancer therapy may lead to cardiovascular complications and can promote each aspect of cardiac disease manifestation, such as vascular disease including coronary heart disease, myocardial diseases including heart failure, structural heart diseases including valvular heart diseases, and rhythm disorders. All potential complications of cancer therapy onto the cardiovascular system require imaging for diagnostic workup as well as monitoring of therapy. While standardized imaging tests like routine transthoracic echocardiography (TTE) can be sufficient for initial evaluation of patient with symptoms suggestive of cardiovascular diseases, specific imaging techniques including nuclear imaging are available to in detail characterize the underlying pathophysiology and lead to improved patient management. Therefore, the field of imaging in cardio-oncology is rapidly growing. While the same imaging modality should be used for continued screening throughout cancer treatment and switching between modalities is strongly discouraged according to current recommendations (1), selection of the appropriate imaging modality has relevant impact on the patient management. This review article will give an overview about existing literature regarding the role of imaging in the diagnostic evaluation and management of therapy in patient with prior or ongoing cancer therapy, providing indications for each imaging technology, stratifying by suspected cardiovascular complication. 


\section{Echocardiography}

Echocardiography is the clinical working horse as leading imaging based diagnostic tool for cardiovascular diseases. Echocardiography is broadly available and frequently used as first imaging modality for checkup-examinations and evaluation of suspected cardiovascular disorders. Given its non-invasive nature with no harm applied to the patient as well as its relatively low costs compared to other imaging modalities, serial echocardiography examinations are used to detect any changes in cardiovascular function during cancer therapy. Moreover, echocardiography not only allows for the display of cardiac structures and function, but also enables assessment of hemodynamics when utilizing Doppler based measures. With the advent of modern echocardiographic techniques including tissue Doppler, Speckle tracking, and three-dimensional echocardiography, indications for echocardiography examinations increased. TTE, where the probe is placed on the chest from the outside, is most frequently preformed in cardio-oncology. Plenty of standardized measures have been established for TTE to evaluate all potential cardiovascular disorders observed in cancer patients. However, image quality may vary based on the patient's habitus and anatomic conditions (e.g., lung emphysema, funnel chest). Moreover, TTE is dependent on the experience of the examiner, making standardized protocols indispensable. In addition to TTE, transesophageal echocardiography (TEE), where the probe is placed in the patient's esophagus, allows more distinct evaluation of the valves and the atria. However, transesophageal echo makes sedation of the patient necessary in many cases, which limits its use in daily practice to specific indications.

\section{Echocardiography for evaluation of vascular diseases}

For assessment of coronary artery disease, echocardiography is frequently performed as initial test to depict cardiac function and rule out other cardiac diseases that may cause the patient's complaints. Normal resting echocardiography examinations may not preclude relevant coronary artery disease, as regional wall motion defects in the setting of coronary artery disease are only observed in critical ischemia or prior myocardial infarction. Instable angina suggestive of coronary artery disease, stress echocardiography, either with pharmacological or physical stress, is widely accepted in clinical practice. There is a tremendous amount of data from large scale, multicenter, effectiveness studies, well demonstrating the safety and prognostic value of stress echocardiography in clinical routine $(2,3)$. Stress echocardiography has improved sensitivity and specificity as compared to exercise electrocardiography and yields the greatest incremental diagnostic and prognostic value in patients in whom exercise electrocardiography is a nondiagnostic, unclear, or inconclusive. Especially in patients in whom exercise is not feasible or contraindicated, pharmacological stress echocardiography can be performed, if imaging quality is sufficient. The results of stress echocardiography can be used as a gatekeeper to coronary angiography. In case of ischemia documented on stress echocardiography in contrast, the prognostic benefit of revascularization therapy on interventional angiography can be increased. Within positive stress echocardiography exams, wall motion defects occurring at low dose or workload, with slow recovery and/or antidote resistance, and effecting more than five segments of the left ventricle are regarded as high risk, and therefore are recommended to receive definite clarification via conventional coronary angiography (4). Combining stress echocardiography with modern echo technologies including speckle tracking and three-dimensional echocardiography further improves diagnostic accuracy.

\section{Echocardiography for evaluation of myocardial diseases}

Echocardiography is the imaging modality of first choice for the detection of myocardial dysfunction before, during, and after cancer therapy $(5,6)$. The biplane twodimensional Simpson's method is predominantly used for estimation of left ventricular ( $\mathrm{LV}$ ) volumes and ejection fraction. If technical requirements are present and image quality is sufficient for clear endomyocardial boarder detection, three-dimensional echocardiography even outperforms tow-dimensional assessment of LV volume and function. For the echocardiography-derived estimation of left ventricular ejection fraction (LVEF), cancer therapeutics related cardiac dysfunction is defined as a decrease in LVEF of $>10$ percentage points to a value below the lower limit of normal $(5,7)$. In case of a decrease in LVEF, repeated assessment is warranted to confirm the impairment in LV systolic function and assess potential reversibility $(1,5)$. A major limitation of the quantification of LVEF by two-dimension al echocardiography is its relatively modest reproducibility, which can be 

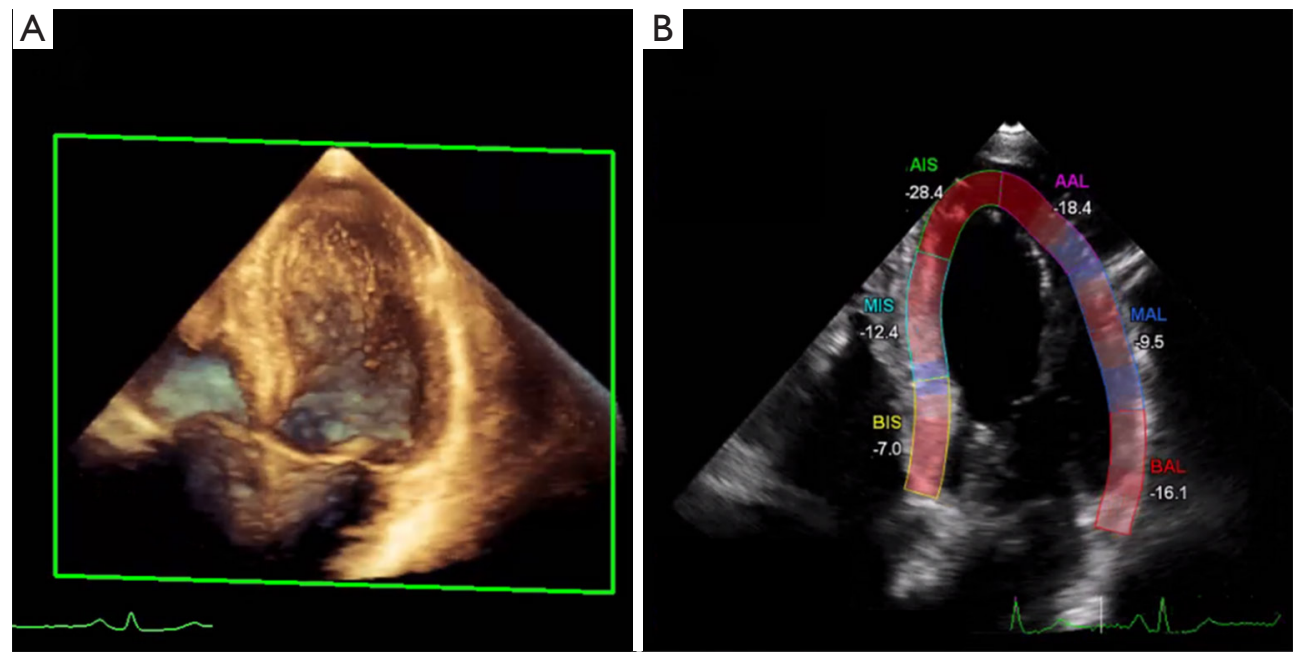

Figure 1 Echocardiographic assessment of a 63 years old female patients following anthracycline based therapy for breast cancer. While left ventricular ejection fraction was normal (3D-LVEF: 61\%), myocardial strain analysis depicted reduced global longitudinal deformation (LV-GLS: $-14.5 \%$ ) as early sign of chemotherapy induced left ventricular dysfunction. LVEF, left ventricular ejection fraction; GLS, global longitudinal strain; AIS, apical inferoseptal; AAL, apical anterolateral; MIS, mid inferoseptal; MAL, mid anterolateral; BIS, basal inferoseptal; BAL, basal anterolateral.

improved using three-dimensional assessment (8). To reduce variability between serial echocardiographic exams over time, current recommendations advise the evaluation of LVEF to be ideally performed by the same observer with the same equipment (5). If image quality is relevantly impaired, the use of echo contrast can improve delineation of the LV endocardial borders (9). As an early marker of LV impairment, deformation imaging via speckle tracking has become clinical standard for serial evaluation in patients undergoing cancer therapy (10). Global systolic longitudinal strain has the ability to predict a subsequent decrease in LVEF (Figure 1) (11,12). Current recommendations describe the relative reduction of $>15 \%$ in global longitudinal strain as abnormal and therefore as marker of subclinical LV dysfunction (1). When available and sufficient expertise at the echo laboratories is present, speckle tracking should be performed to serve as the basis for clinical decisions (13). In addition to global strain, also regional strain and three-dimensional strain have been introduced as potential early markers with promising results $(14,15)$. As three-dimensional strain analysis may warrant the ability to assess the full volume deformation, it reduces errors that can occur with two-dimensional imaging (16).

In addition to systolic impairments, diastolic dysfunction is a common finding in cancer patients at baseline and under treatment. Diastolic dysfunction, such as prolonged isovolumic relaxation period, a reduction in peak flow velocity, the ration of early peak flow velocity to atrial peak flow velocity, and a reduction in the deceleration rate of the early peak flow velocity can precede resting systolic dysfunction $(17,18)$. Stoddard et al reported that prolongation of isovolumic relocation time by Doppler echocardiography predicts systolic dysfunction in patients receiving doxorubicin (18). A doxorubicininduced reduction in ejection fraction of $>10 \%$ to $<55 \%$ was observed in 9 out of 26 patients of this study with the isovolumic relaxation time increasing from 66 to $84 \mathrm{~ms}$ after a cumulative doxorubicin dose of 100 to $120 \mathrm{mg} / \mathrm{m}^{2}$. However, the modest reproducibility of diastolic measures from echocardiography limits its value with diastolic measures having varying levels of success in identifying signs of impaired cardiac function following cancer therapy $(19,20)$. Therefore, so far there is not data, advising that cancer treatment should be altered based on diastolic indices.

\section{Echocardiography for evaluation of structural heart diseases}

While chemotherapeutic agents do not directly affect cardiac valves, valvular heart disease is frequently observed in patients with cancer due to radiotherapy, infective 
endocarditis, pre-existing valve lesion, or secondary to $\mathrm{LV}$ dysfunction $(5,21,22)$. Approximately $10 \%$ of patient treated with radiation develop a relevant valvular heart disease during lifetime (23). As radiation induced valvular heart disease shows a distinct pattern with fibrosis and calcification of the aortic root, the aortic valve cusps, mitral valve annulus, and the base and mid portions of the mitral valve leaflets, sparing the mitral valve tips and commissures, echocardiography may be capable of differentiating it from rheumatic disease $(21,23)$. In patients with Hodgkin lymphoma, a relationship between radiation dose to the heart and development of valvular events after treatment has been described, especially at doses >30 Gy (24). However, as mediastinal radiation dose is limited to 20 or 30 Gy in today's protocols, the 30-year risk for valvular heart disease only modestly increases compared to patients never receiving radiation therapy (25). Echocardiography is the imaging method of choice for suspected valvular heart disease and for follow-up examinations after cancer therapy $(1,26)$. While two-dimensional TTE is sufficient to rule out relevant valvular diseases, transesophageal echocardiography utilizing three-dimensional acquisitions may be mandatory to allow for a more distinct description of the underlying pathology, especially in disorders of the mitral valve.

\section{Echocardiography for evaluation of rhythm disorders}

Echocardiography is suggested in patients with rhythm disorders to detect underlying structural heart diseases. In the case of atrial fibrillation, this especially includes the assessment of left and right atrial size as well as assessment of tricuspid and mitral value function. In addition, echocardiography allows the assessment of measures of increased ventricular filling pressure, which can also lead to atrial enlargement and hence may cause atrial rhythm disorders. In case of ventricular arrhythmias, echocardiography can detect underlying pathologies, which may include myocardial scar and hypertrophic or dilative cardiomyopathy. Likewise, echocardiography can be used for suspected rare causes of ventricular arrhythmias including cardiac amyloidosis, arrhythmogenic right ventricular cardiomyopathy, or cardiac sarcoidosis, while further work-up in a multi-imaging approach may be mandatory.

In symptomatic patients with nonvalvular atrial fibrillation, the sinus rhythm can be restored by pharmacological or electrical cardioversion (27). The periprocedural risk of thromboembolic events in non- anticoagulated patients is $5-7 \%$, with a higher risk for patients with heart failure and diabetes mellitus (28-30). The predominant risk of thromboembolic events arises from potential thrombi located in the left atrium and most importantly in the left atrial appendage. Transesophageal echocardiography can exclude the appearance of atrial thrombi prior to cardioversion (31). Therefore, the utilization of transesophageal echocardiography can allow for direct cardioversion in patients with atrial fibrillation with more than 48 hours onset, as otherwise the actual guidelines recommend the use of oral anticoagulants at least 3 weeks prior to cardioversion.

\section{Cardiac computed tomography (CT)}

Over the last decade, indications for cardiac CT have increased. Non-contrast CT exams are predominantly used for quantification of subclinical coronary atherosclerosis to improve risk prediction in appropriate populations (32). Contrast enhanced exams, however, are frequently used for rule out of coronary artery disease in patients presenting with chest pain, due to its high negative predictive value (33). For patients during or following cancer therapy, indications for CT imaging may alter, due to the specific circumstances in this cohort.

\section{Cardiac CT for evaluation of vascular diseases}

Coronary CT angiography enables assessment of coronary plaque burden and cardiovascular disease manifestation (34). The predominant clinical indication of coronary CT angiography arises from its high negative predictive value, allowing for a safe and time-effective rule out of stenotic coronary artery disease in a majority of symptomatic patients with low- to intermediate pretest probability of coronary artery disease $(33,35,36)$. Following radiation therapy, there is a dose dependent association with development with vascular diseases (37). Traditional cardiovascular risk factors may even magnify the risk of cancer therapy with a latency period of 10 years after the initial cancer therapy (38). As presentation of coronary artery disease may frequently be atypical and the prevalence of silent ischemia is higher than in other coronary artery disease cohorts (39), coronary CT angiography may be an ideal test for disease assessment in suspected disease despite the lack of typical symptoms. For patients following treatment for testicular cancer, a more than two-fold increased risk of coronary artery disease manifestation 10 years after treatment is 
observed (40). When receiving both multidrug cisplatinbased chemotherapy in combination with radiation therapy, the absolute risk for ischemic events 20 years after treatment may increase up to $8 \%$ (41). Likewise, an increased risk for sudden cardiac death has been reported due to intimal hyperplasia of all coronary arteries, including the left main, for cancer patients even without radiation therapy (42). Therefore, in the case of typical presentation suggestive of underlying coronary artery disease in patients after cancer therapy, pretest-likelihood may be too high to advocate for the use of coronary CT angiography.

For patients prior to initiation of cancer treatment, identification of patients with pre-existing coronary artery disease is crucial, as pre-existing subclinical coronary atherosclerosis substantially increases the risk of developing treatment related clinical coronary artery disease manifestation (6). In addition, patients who develop an acute coronary syndrome or symptoms suggestive of a coronary artery disease while thrombocytopenic during chemotherapy reflect a particular challenge for treatment. In these cases, cardiac CT may be an alternative to conventional coronary angiography for assessment of presence and extent of disease to improve future patient management. If obstructive disease can be ruled out via coronary CT angiography, subsequent antithrombotic therapy, leading to an increased bleeding risk, may be avoided (43).

For asymptomatic patients, non-contrast enhanced cardiac CT for quantification of the coronary artery calcification score has the ability to assess the individuals' risk for future cardiovascular events (44). Estimation of coronary artery calcification via non-contrast CT of the heart is the best single existing test to improve prediction and reclassification of asymptomatic patients $(45,46)$. As patients after cancer therapy may be asymptomatic due to neurotoxicity of radiotherapy or chemotherapy, affecting the patient's perception of angina, an imaging-based test of subclinical atherosclerosis may improve the estimation of the individual risk and direct towards improved primary prevention algorithms (47). However, dedicated studies on patients following cancer therapy to predict long term cardiovascular outcome need to confirm the results form population-based cohort studies.

\section{Cardiac CT for evaluation of myocardial diseases}

Generally, assessment of ventricular sizes and function is feasibly, however, especially for function analysis, radiation exposure throughout the complete cardia cycle is mandatory, while its additional value over echocardiography and especially magnetic resonance imaging (MRI) regarding myocardial evaluation is limited. Comparing to the ability of MRI to assess ventricular sizes and function without applying radiation to the patients and to additionally detect myocardial edema or signs of myocardial injury, cardiac CT for assessment of myocardial diseases may only be limited to patients with contra-indications to MRI and insufficient image quality on echocardiography. For both contrasts enhanced and non-contrast CT, standardized measures have been described to enable reliable quantification of atrial and ventricular dimensions (48-50).

\section{Cardiac CT for evaluation of structural heart diseases}

In case of valvular heart diseases, CT may be useful for detecting the extent of calcifications of the ascending aorta as frequently seen after mediastinal radiation therapy, which may relevantly increase the operative risk and sometimes prohibits conventional open-heart surgery (51). Also, in case of suspected low-flow low-gradient aortic valve stenosis in patients with impaired LV function, quantification of valvular calcification may help to distinguish true aortic stenosis requiring specific therapy from pseudo lowflow low-gradient stenosis (52). In case of interventional trans-femoral or trans-apical aortic valve implantation, a cardiac CT scan is mandatory for sizing of the aortic annulus, which should include CT imaging of the aorta and femoral arteries, if a trans-femoral access is preferred (36). In addition, CT of the heart is preferred for assessment of changes in the pericardium in suggested pericardial diseases, which can follow radiation or chemo therapy or combination of both. CT scan depict both calcification and thickening of the pericardium $(53,54)$. If a pericardial effusion is present, three-dimensional assessment via CT enables reliable quantification of the extent and can differentiate between hemorrhagic and serous effusion (55).

\section{CT for evaluation of rhythm disorders}

For rhythm disorders, there is only a limited indication for cardiac CT. For prospectively gated scanning, which represents the majority of CT examinations, atrial fibrillation and atrial flutter relevantly impairs the image acquisition. Likewise, CT scanning may be complicated, if the patient suffers from tachycardia, especially, when single source scanners are used. In contrast, if a stable 
heart rhythm is achieved, coronary CT angiography can be used for detection of suspected underlying coronary artery disease. Moreover, cardiac CT allows for three-dimensional imaging of the left atrium and the pulmonary veins can be of use in the planning of electrophysiological examinations while when integrated in the fluoroscopic image can improve procedural success. After pulmonary vein ablation, CT of the heart is the gold standard for detection of a pulmonary vein stenosis $(56,57)$.

\section{MRI}

MRI has developed as a powerful tool for cardiovascular imaging over the last decades. For the work-up of different diseases it is increasingly accepted as the mainstay due its large variety of imaging capabilities which include the quantification of right and LV systolic function, the assessment of wall motion abnormalities and for detailed tissue characterization (such as fibrosis, scarring, fat or edema). Other major advantages of MRI include the very high soft-tissue contrast and the lack of ionizing radiation to the patient. Also, many patients are able to receive Gadolinium-based contrast agents except for patients with severe kidney failure, while many patients do not tolerate contrast agents for CT. Major drawbacks of MRI are, however, the high magnetic fields (particularly 3 Tesla and above) which do often not allow to image patients with various cardiac devices such as pacemakers, internal cardiac defibrillators, left-ventricular assist devices or mechanical heart valves. It is also important to note that MRI may not be performed in patients with certain breast tissue expanders containing ferromagnetic components (58). Nevertheless, due to its big advantages, cardiac MRI is recognized as a tool for the identification of cardiovascular dysfunction caused by cancer-related treatment by the American College of Cardiology/American Heart Association (59).

\section{Detection of coronary artery disease and early vascular damage}

Cardiac MRI in combination with pharmacological stress testing is able to detect coronary artery disease and quantify the extent of ischemia with high diagnostic accuracy (60). Cardiac MRI for the detection of coronary artery disease can be performed visually, quantitatively, and semi-quantitatively. With visual interpretation the reader identifies regions of the heart with delayed perfusion, i.e., delayed signal brightening during contrast application. For semiquantitative assessment an upslope index is usually calculated between the stress and the rest studies for the different myocardial segments. Absolute quantification of myocardial perfusion with MRI is challenging as it is mathematically highly complex, applies different kinetic analysis methods (e.g., Fermi-constrained deconvolution) and its accuracy is still a matter of debate $(61,62)$. Consequently, cardiac MRI may be used to rule out coronary artery disease before the initiation of cancer treatment. Theoretically, cardiac perfusion MRI could potentially be used to detect perfusion deficits occurring during or after cardiotoxic treatment regimens for cancer, however, no larger trials are available. On the other hand, it would also be of high clinical impact, if MRI would allow for assessment of early vascular toxicity and damage before flow-limiting disease occurs. One way to identify vascular changes at a subclinical stage is the assessment of pulse wave velocity (PWV). PWV is a marker that is directly related to vessel stiffness, which in turn allows to draw conclusions on atherosclerotic disease burden in an early stage (63). MRI allows to measure both the local and central arterial stiffness. After the administration of cardiotoxic chemotherapy such as anthracyclines, the PWV of the thoracic aorta - as an indicator of vascular stiffnessincreases steadily. This phenomenon and also the potential of reversibility of these early vascular changes after discontinuation of anthracyclines has been demonstrated in breast cancer patients (64). Another finding that has been described is that arterial stiffness is increased in patients that have survived cancer in childhood after chemotherapy (65).

\section{Assessment of treatment-induced myocardial damage}

Using cardiac MRI early changes in the myocardium, induced by cancer-related treatments, can be assessed. Parameters include but are not restricted to the assessment of edema (T2-weighted imaging), fibrosis and extracellular volume (native and post-contrast T1 mapping), and scarring (late gadolinium enhancement). As these biomarkers mostly indicate early changes in the heart before the occurrence of $L V$ systolic function impairment, the assessment of these parameters is of high clinical relevance for monitoring myocardial health during cardiotoxic cancer-treatments. Myocardial edema is thought to be one of the earliest surrogate markers of chemotherapy or radiation induced damage to the heart. In a study by Oberholzer et al., 28 patients receiving anthracycline-based chemotherapies underwent cardiac 
MRI (66). In 2 patients signal alterations on T2-weighted images suspicious for myocardial edema were detected. In another study 46 patients receiving chemotherapy regimens of either anthracycline alone or in combination with trastuzumab for breast cancer were studied using cardiac MRI (67). On average taking all patients into account, the authors found an increase in myocardial edema after starting chemotherapy and this observation lasted for 4 months. When using a certain binary cutoff $33 \%$ and $49 \%$ of patients were diagnosed to have myocardial edema after 1 and 4 months, respectively. Interstitial myocardial fibrosis caused by collagen accumulation has also been observed after different chemotherapy regimen including anthracycline, trastuzumab and also after radiation therapy $(68,69)$. Collagen depositions are thought to be one reason for impairment of LV function after cancertreatment. Consequently, beginning of collagen deposition precedes impaired $L V$ function and is a very interesting early biomarker of myocardial damage. Of note, other cardiovascular diseases or risk factors may be the reason of collagen deposition including myocarditis, myocardial infarction, hypertension, or diabetes. In a study on 30 children with imaging using cardiac MRI 2 years following anthracycline therapy was performed (68). It was found that despite having a normal LVEF, alterations in parameters including extracellular volume fraction (ECV) and T1 values were found that correlated with cumulative dose of chemotherapy, exercise capacity and myocardial wall thinning, indicating that early myocardial damage may be assessed using cardiac MRI. With the late gadolinium enhancement technique focal fibrosis/scarring can be visualized using cardiac MRI. Data on the occurrence of focal late gadolinium enhancement (LGE) after cancerrelated treatment is inconclusive. While LGE is reported as a frequent finding with a prevalence of $25 \%$ in patients after trastuzumab therapy in one study (70), another study reported an incidence of only $6 \%$ in patients treated with anthracycline-based chemotherapy (71). Further studies on the occurrence and significance of LGE after cardiotoxic, anti-cancer treatments are warranted.

\section{Detection of myocardial dysfunction}

Cardiac MRI is often referred to as the method of reference for the quantification of LV volumes, mass, and wall motion abnormalities (72). Still, MRI is usually not the first choice for risk stratification prior to initiation of cardiotoxic treatment for cancer. While echocardiography is usually performed first, MRI may help in case of nondiagnostic studies, in patients with unclear dilatation of the right or left ventricle, or if there is a concern for an infiltrative cardiac disease. Still, no data is available, if MRI performed prior to cardiotoxic treatment is able to identify patients who are at a higher risk. MRI is often applied for the assessment of LVEF with the aim to identify LV dysfunction after anti-cancer treatment (58). Usually, LVEF is calculated from short-axis slices, covering the whole heart using steady-state free precession sequences. Several studies are available that documented a decline in LVEF during, 12 or 24 months after anthracycline-based chemotherapy $(70,73,74)$. Interestingly, in a study by Wassmuth et al., a decline in LVEF was found to occur as early as 1 month after the initiation of anthracycline-based chemotherapy at a time when patients received only 1 to 2 doses of chemotherapy (74). Also, increases in LV endsystolic volumes have been described particularly with anthracycline-based chemotherapy or with trastuzumab (70,73). More recently, LV global longitudinal strain has been used to identify early LV dysfunction (75). In a cardiac MRI study 53 patients with breast cancer, leukemia or lymphoma receiving low to moderate doses of anthracyclinebased chemotherapy were studied regarding changes in parameters such as $\mathrm{LV}$ volumes, LVEF, circumferential strain or late gadolinium enhancement (73). Within the first 6 months after initiation of chemotherapy a significant increase in LV end-systolic volume and strain was found, while a decrease of LVEF was described-findings that are associated with the future occurrence of cardiovascular events. Another important and easily accessible parameter of the heart is the LV mass. In a study by Neilan et al., 91 patients with a cardiomyopathy caused by anthracycline-based chemotherapy were followed up for 27 months and studied using cardiac MRI to identify factors associated with cardiovascular events such as cardiovascular death, ICD implantation, and admission of decompensated heart failure (71). Despite a reduced LVEF, late gadolinium enhancement was an uncommon finding $(6 \%$ of patients only). The LV mass quantified by cardiac MRI, however, was inversely correlated with the anthracycline dose. Furthermore, LV mass was the strongest prognostic factor regarding the occurrence of major cardiovascular events. In this study, an indexed $\mathrm{LV}$ mass of $\leq 57 \mathrm{~g} / \mathrm{m}^{2}$ yielded a sensitivity of $100 \%$ and a specificity of $85 \%$ to predict major cardiovascular events.

In conclusion, cardiac MRI is a versatile tool that gives access to a large number of parameters that are known to be associated with future cardiac events. Cardiac MRI 
thus allows risk stratification of patients prior to or during cancer-related treatments without any radiation dose. However, patients with cardiac devices such as pacemakers or ICDs and patients with renal insufficiency may often not be imaged using this modality. In those patients, alternative imaging strategies such as CT or nuclear medicine techniques should be considered.

\section{Nuclear cardiac imaging}

In nuclear medicine radiotracers are used at pico- or nanomolar concentrations not affecting the studied target such as enzymes, receptors or metabolic pathways. As a consequence, a myriad of targets is available that can be studied non-invasively (71). The biggest advantage of nuclear medicine imaging is the high sensitivity, while-at the same time-one of the major drawbacks is the limited spatial resolution. Positron emission tomography (PET) and single photon emission computed tomography (SPECT) scanners are usually combined with a CT-scanner in order to allow co-registration of the molecular signal with anatomical information and for the purpose of attenuation correction. The newest generation of scanners in nuclear medicine are hybrid PET/MRI-scanners allowing the simultaneous acquisition of PET and MRI (76). As most used radiotracers have a rather short half-live (e.g., Tc-99m: 6 hours, F-18: 110 minutes) radiation dose to the patient is acceptable and is even decreasing with novel camera technology (e.g., cadmium-zinc-telluride crystals) (77). As a consequence, nuclear medicine techniques are well-suited for the identification of early alterations of the myocardium after cancer-related, cardiotoxic treatments.

\section{Assessment of LV function}

As described above, $L V$ dysfunction is a very common finding in patients suffering from cardiotoxicity. While techniques such as echocardiography and MRI are increasingly utilized to estimate $\mathrm{LV}$ volumes and systolic function, nuclear medicine techniques such as multigated radionuclide angiography in 2-dimensional (MUGA, syn. radionuclide ventriculography) or 3-dimensional [also referred to as gated blood-plood SPECT (GBPS)] have been used for this purpose for a long time. This technique is highly reproducible and may also be performed in patients with cardiac devices (not compatible with MRI) or in patients in whom echocardiography is difficult to perform (e.g., obese subjects) (78). A multitude of studies that demonstrated the value of this technique have been published. In a study by Ewer et al. published in the Journal of Clinical Oncology, 38 patients with breast cancer and the suspicion of trastuzumab-related cardiotoxicity were studied using MUGA (79). The authors found a significant decrease of LVEF during trastuzumab treatment that recovered in 37 of 38 patients. The mean time to LVEF recovery was 1.5 months. Even though acceptable, radiation dose is the main reason that MUGA has been replaced by MRI or echocardiography in many cases. Also, myocardial perfusion imaging in gated technique allows a highly accurate estimation of the LV systolic function and has therefore been increasingly used (80).

\section{Myocardial perfusion imaging (MPI)}

In nuclear cardiology, PET and SPECT are still most often performed for the assessment of myocardial perfusion, particularly for clinical questions such as diagnosis of coronary artery disease, hemodynamic significance of a coronary stenosis, for therapy guidance (e.g., extent of myocardial ischemia), and for risk stratification of patients (76). While SPECT MPI is the most widely available technique for this purpose with millions of scans in the US every year (81), PET allows for absolute quantification of regional myocardial blood flow. With regards to myocardial perfusion imaging the majority of studies focused on patients that underwent radiation therapy because of breast or thoracic cancers. In a study by Gayed et al., 51 patients with esophageal cancer were studied regarding myocardial perfusion defects (82). A total of 26 patients underwent radiation therapy and $54 \%$ of those patients demonstrated myocardial perfusion defects. In the group that did not undergo radiation $(\mathrm{n}=25)$, only $16 \%$ had perfusion defects. LVEF did not differ between the two groups. Interestingly, 11 of the 14 patients had inferior wall ischemia and all of these patients had distal esophageal cancer indicating a direct relationship between radiation therapy and occurrence of local ischemia (Figure 2). In another study breast cancer patients who were randomized to conventional chest wall radiation or to radiation using a so-called active breath coordinator (ABC) were studied regarding the occurrence of myocardial perfusion defects (83). With $\mathrm{ABC}$ radiation is delivered when the heart is farther from the irradiation area which in turn reduces cardiac radiation exposure. The hypothesis that 

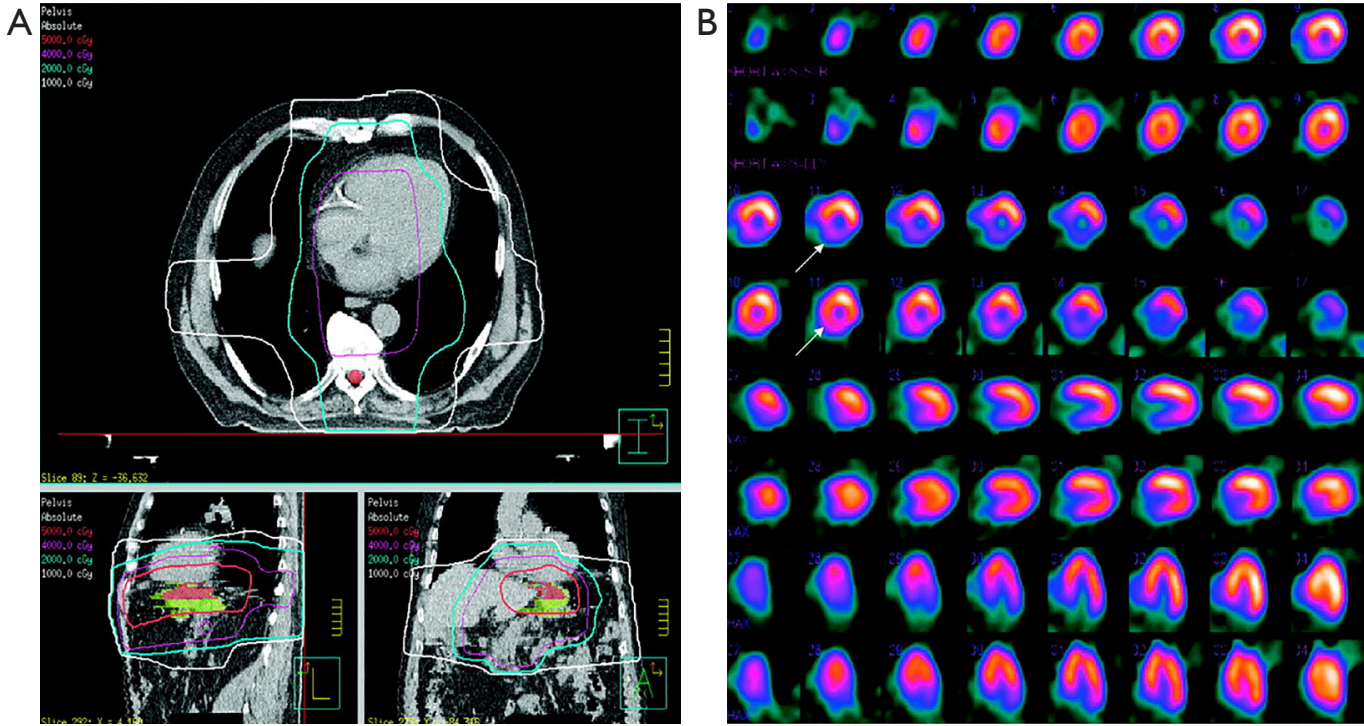

Figure 2 Planning CT of a patient with esophageal cancer who had to undergo radiation (A: planning CT with isocontours of radiation doses). Myocardial perfusion images demonstrate ischemia of the inferior wall (B: white arrows), that was included in the 4,000-5,000 cGy isocontour. (This research was originally published in $7 N M$. Gayed IW, Liu HH, Yusuf SW, Komaki R, Wei X, Wang X, Chang JY, Swafford J, Broemeling L and Liao Z, J Nucl Med. 2006;47:756-62. (CSNMMI).

ABC-guided radiation would reduce perfusion defects had to be rejected, however, as perfusion defects were found at a similar frequency in the apex and the anterior wall in both groups. While PET studies on myocardial perfusion imaging in patients undergoing cardiotoxic treatment are scarce and delivered controversial results $(84,85)$, an experimental study in dogs that underwent local heart irradiation clearly demonstrated myocardial perfusion defects on N-13 ammonia PET/CT (86). Interestingly, the irradiated dogs' hearts demonstrated an initial increase in perfusion after 3 months, a reduced perfusion after 6 months and a perfusion defect after 12 months. Furthermore, LVEF decreased over time in irradiated dogs.

\section{Assessment of myocardial damage}

Radiotracers are available that allow the visualization of cell apoptosis or necrosis. Tc-99m-annexin $\mathrm{V}$ is a radiotracer that binds to phosphatidylserine, a molecule that is exposed on the membrane of cells undergoing apoptosis. The concept to image apoptosis as a surrogate marker of cardiotoxicity due to doxorubicin has been successfully demonstrated in a rodent study (87). It has also been demonstrated that radiotracer uptake correlates with the grade of toxicity on histopathology $(88,89)$. Another tracer to image myocardial injury is In-111-antimyosin. As this In-111-labeled murine monoclonal anti-myosin Fab antibody fragment can only bind to intracellular myosin when the sarcolemma membrane is disrupted, it is a marker of cellular necrosis. This tracer has been used in several studies to investigate cardiotoxicity of chemotherapy (90-93). Using this tracer, the dose-dependent cardiotoxicity of epirubicin has been demonstrated in cancer patients. In this study, it has also been demonstrated that a higher intensity of tracer uptake, reflecting a higher degree of necrosis in the heart, was associated with an impairment of LV systolic function in these patients (94). However, the specificity of In-111-antimyosin uptake as a prognostic marker for the development of heart failure has been questioned by a study, where long-term effects of anthracyclines (up to 10 years after chemotherapy) were studied (95). In this study both patients with recovery of LVEF and patients with a persistent decrease of LVEF showed an increased radiotracer uptake. With regards to radiation induced myocardial necrosis only limited experience with In-111 anti-myosin scintigraphy is available (96).

\section{Metabolic and innervation alterations as an early marker of cardiotoxicity}

The most often applied tracer for PET imaging is F-18 fluorodeoxyglucose (FDG). F-18 FDG is a glucose 
analogue and is taken up via glucose-transporters into the cells. Depending on the preparation of the patient, the cardiomyocyte either prefers glucose or free fatty acid as primary substrate (97). If viability imaging is performed, the patient receives-depending on the used protocoleither a drink with high glucose content (glucose loading) in order to enhance myocardial F-18 FDG uptake or the hyperinsulinemic euglycemic clamp technique is performed. In order to image myocardial inflammation, the cardiomyocytes glucose consumption is minimized by different strategies: high-fat low-carb diet the prior to F-18 FDG PET, prolonged fasting or pre-injection of heparin before F-18 FDG administration (the latter stimulates various lipases which increases free fatty acid levels in the blood). If F-18 FDG is injected with such a preparation, the physiological F-18 FDG accumulation in the heart is minimized and inflammatory foci may be imaged with high contrast (98). In a preclinical study in mice treated with the tyrosine kinase inhibitor sunitinib, an enhanced glucose uptake of the heart was found 48-72 hours after initiation of the treatment (99). Furthermore, the animals demonstrated a decrease of LVEF as a sign of beginning heart failure. In addition, ultrastructural changes such as lipid accumulation and perturbed mitochondrial function were found. Also, a case report describes the cardiotoxicity of a combination of tyrosine kinase inhibitors (sorafenib and imatinib) using F-18 FDG PET in a patient dying of heart failure (100). Accumulating evidence suggests that chemotherapy with anthracyclines alters the myocardial glucose metabolism as evidenced by F-18 FDG PET. In preclinical and retrospective clinical studies, an increase in F-18 FDG accumulation in the heart was observed as a potential early sign of cardiotoxicity (101-104). Besides chemotherapyinduced cardiotoxicity, radiation-related myocardial metabolic alterations have been described in a preclinical study in dogs (105). Concordantly, in a retrospective clinical study, an increased F-18 FDG uptake of the heart was found after stereotactic radiation of lung tumors close to the heart (106). The cardiac F-18 FDG uptake correlated with radiation dose to the heart possibly indicating radiationinduced myocardial damage.

Another radiotracer that was frequently used to image early signs of cardiotoxicity is I-123 metaiodobenzylguanidine (MIBG), a SPECT tracer. Usually, MIBG scintigraphy is performed early, i.e., 15 minutes, and late, i.e., 4 hours after tracer injection. Using this protocol, the heart-tomediastinum ratio (a parameter of neuronal function) and the washout-rate (a marker of sympathetic tone) can be calculated (107). Sympathetic nerve fibers are more sensitive than cardiomyocytes with regards to noxae such as ischemia or cardiotoxic agents. As a consequence, first signs of cardiotoxicity due to chemotherapy or radiation may be found on MIBG before the occurrence of impairment of LV systolic function. Preclinically, the concept has been proven e.g., using I-125 MIBG in a rat model of Adriamycin-induced cardiomyopathy (108). Subsequently, it has been demonstrated that I-123 MIBG can be used to image detrimental effects of an anthracycline-based therapy in humans and that the decrease in I-123 MIBG shows a dose-dependency $(109,110)$. Alternative PET radiotracers to image sympathetic innervation of the heart are available (e.g., C-11 meta-hydroxyephedrine, C-11 phenylephrine, C-11 epinephrine) but not widely used (111). So far, however, only very limited preclinical data and no studies in humans are available (112).

\section{Summary}

A large armamentarium of imaging modalities is available which may be used to monitor cardiotoxic effects of anticancer treatments. The big advantage of echocardiography is that it is widely available, cheap and operates without ionizing radiation. CT is very well-suited for the assessment of coronary anatomy. MRI is useful to detected myocardial perfusion abnormalities, scarring and early signs of myocardial damage such as edema and beginning fibrosis. Nuclear medicine allows to image molecular signals of myocardial damage such as perfusion abnormalities, cell death and alterations in metabolism and innervation. Most studies and imaging modalities focus on impairments of $\mathrm{LV}$ function and wall motion, and thus already at a late state in the process of cardiotoxicity. Recently, however, novel approaches to identify early prognostic factors before the occurrence of LV function impairment particularly using cardiac MRI and nuclear medicine techniques are investigated. With novel treatments (e.g., checkpoint inhibitor therapy), close monitoring of patients in order to identify possible cardiotoxicity is essential and prospective studies to investigate surrogate markers of occurring cardiotoxicity are warranted.

\section{Acknowledgements}

None. 


\section{Footnote}

Conflicts of Interest: The authors have no conflicts of interest to declare.

\section{References}

1. Zamorano JL, Lancellotti P, Rodriguez Munoz D, et al. 2016 ESC Position Paper on cancer treatments and cardiovascular toxicity developed under the auspices of the ESC Committee for Practice Guidelines: The Task Force for cancer treatments and cardiovascular toxicity of the European Society of Cardiology (ESC). Eur Heart J 2016;37:2768-801.

2. Picano E, Mathias W Jr., Pingitore A, et al. Safety and tolerability of dobutamine-atropine stress echocardiography: a prospective, multicentre study. Echo Dobutamine International Cooperative Study Group. Lancet 1994;344:1190-2.

3. Picano E, Sicari R, Landi P, et al. Prognostic value of myocardial viability in medically treated patients with global left ventricular dysfunction early after an acute uncomplicated myocardial infarction: a dobutamine stress echocardiographic study. Circulation 1998;98:1078-84.

4. Sicari R, Nihoyannopoulos P, Evangelista A, et al. Stress echocardiography expert consensus statement: European Association of Echocardiography (EAE) (a registered branch of the ESC). Eur J Echocardiogr 2008;9:415-37.

5. Plana JC, Galderisi M, Barac A, et al. Expert consensus for multimodality imaging evaluation of adult patients during and after cancer therapy: a report from the American Society of Echocardiography and the European Association of Cardiovascular Imaging. Eur Heart J Cardiovasc Imaging 2014;15:1063-93.

6. Lancellotti P, Nkomo VT, Badano LP, et al. Expert consensus for multi-modality imaging evaluation of cardiovascular complications of radiotherapy in adults: a report from the European Association of Cardiovascular Imaging and the American Society of Echocardiography. Eur Heart J Cardiovasc Imaging 2013;14:721-40.

7. Lang RM, Badano LP, Mor-Avi V, et al. Recommendations for cardiac chamber quantification by echocardiography in adults: an update from the American Society of Echocardiography and the European Association of Cardiovascular Imaging. Eur Heart J Cardiovasc Imaging 2015;16:233-70.

8. Thavendiranathan P, Grant AD, Negishi T, et al. Reproducibility of echocardiographic techniques for sequential assessment of left ventricular ejection fraction and volumes: application to patients undergoing cancer chemotherapy. J Am Coll Cardiol 2013;61:77-84.

9. Suwatanaviroj T, He W, Pituskin E, et al. What is the minimum change in left ventricular ejection fraction, which can be measured with contrast echocardiography? Echo Res Pract 2018;5:71-7.

10. Armenian SH, Hudson MM, Mulder RL, et al. Recommendations for cardiomyopathy surveillance for survivors of childhood cancer: a report from the International Late Effects of Childhood Cancer Guideline Harmonization Group. Lancet Oncol 2015;16:e123-36.

11. Sawaya H, Sebag IA, Plana JC, et al. Assessment of echocardiography and biomarkers for the extended prediction of cardiotoxicity in patients treated with anthracyclines, taxanes, and trastuzumab. Circ Cardiovasc Imaging 2012;5:596-603.

12. Negishi K, Negishi T, Hare JL, et al. Independent and incremental value of deformation indices for prediction of trastuzumab-induced cardiotoxicity. J Am Soc Echocardiogr 2013;26:493-8.

13. Voigt JU, Pedrizzetti G, Lysyansky P, et al. Definitions for a common standard for 2D speckle tracking echocardiography: consensus document of the EACVI/ ASE/Industry Task Force to standardize deformation imaging. Eur Heart J Cardiovasc Imaging 2015;16:1-11.

14. Sawaya H, Sebag IA, Plana JC, et al. Early detection and prediction of cardiotoxicity in chemotherapy-treated patients. Am J Cardiol 2011;107:1375-80.

15. Poterucha JT, Kutty S, Lindquist RK, et al. Changes in left ventricular longitudinal strain with anthracycline chemotherapy in adolescents precede subsequent decreased left ventricular ejection fraction. J Am Soc Echocardiogr 2012;25:733-40.

16. Pizzino F, Vizzari G, Qamar R, et al. Multimodality Imaging in Cardiooncology. J Oncol 2015;2015:263950.

17. Tassan-Mangina S, Codorean D, Metivier M, et al. Tissue Doppler imaging and conventional echocardiography after anthracycline treatment in adults: early and late alterations of left ventricular function during a prospective study. Eur J Echocardiogr 2006; 7:141-6.

18. Stoddard MF, Seeger J, Liddell NE, et al. Prolongation of isovolumetric relaxation time as assessed by Doppler echocardiography predicts doxorubicin-induced systolic dysfunction in humans. J Am Coll Cardiol 1992;20:62-9.

19. Banchs J, Jefferies JL, Plana JC, et al. Imaging for cardiotoxicity in cancer patients. Tex Heart Inst J 2011;38:268-9. 
20. Civelli M, Cardinale D, Martinoni A, et al. Early reduction in left ventricular contractile reserve detected by dobutamine stress echo predicts high-dose chemotherapyinduced cardiac toxicity. Int J Cardiol 2006;111:120-6.

21. Hering D, Faber L, Horstkotte D. Echocardiographic features of radiation-associated valvular disease. Am J Cardiol 2003;92:226-30.

22. Brosius FC 3rd, Waller BF, Roberts WC. Radiation heart disease. Analysis of 16 young (aged 15 to 33 years) necropsy patients who received over 3,500 rads to the heart. Am J Med 1981;70:519-30.

23. Hull MC, Morris CG, Pepine CJ, et al. Valvular dysfunction and carotid, subclavian, and coronary artery disease in survivors of hodgkin lymphoma treated with radiation therapy. Jama 2003;290:2831-7.

24. Cutter DJ, Schaapveld M, Darby SC, et al. Risk of valvular heart disease after treatment for Hodgkin lymphoma. J Natl Cancer Inst 2015;107.

25. Malanca M, Cimadevilla C, Brochet E, et al. Radiotherapyinduced mitral stenosis: a three-dimensional perspective. J Am Soc Echocardiogr 2010;23:108.e1-2.

26. Jaworski C, Mariani JA, Wheeler G, et al. Cardiac complications of thoracic irradiation. J Am Coll Cardiol 2013;61:2319-28.

27. Kirchhof P, Benussi S, Kotecha D, et al. 2016 ESC Guidelines for the management of atrial fibrillation developed in collaboration with EACTS. Eur Heart J 2016;37:2893-962.

28. Stellbrink C, Nixdorff U, Hofmann T, et al. Safety and efficacy of enoxaparin compared with unfractionated heparin and oral anticoagulants for prevention of thromboembolic complications in cardioversion of nonvalvular atrial fibrillation: the Anticoagulation in Cardioversion using Enoxaparin (ACE) trial. Circulation 2004;109:997-1003.

29. Airaksinen KE, Gronberg T, Nuotio I, et al. Thromboembolic complications after cardioversion of acute atrial fibrillation: the FinCV (Finnish CardioVersion) study. J Am Coll Cardiol 2013;62:1187-92.

30. Hansen ML, Jepsen RM, Olesen JB, et al. Thromboembolic risk in 16274 atrial fibrillation patients undergoing direct current cardioversion with and without oral anticoagulant therapy. Europace 2015;17:18-23.

31. Kirchhof P, Benussi S, Kotecha D, et al. 2016 ESC

Guidelines for the management of atrial fibrillation developed in collaboration with EACTS. Eur J Cardiothorac Surg 2016;50:e1-88.

32. Mahabadi AA, Mohlenkamp S, Moebus S, et al. The
Heinz Nixdorf Recall study and its potential impact on the adoption of atherosclerosis imaging in European primary prevention guidelines. Curr Atheroscler Rep 2011;13:367-72.

33. Hoffmann U, Truong QA, Schoenfeld DA, et al. Coronary CT angiography versus standard evaluation in acute chest pain. N Engl J Med 2012;367:299-308.

34. Nielsen LH, Botker HE, Sorensen HT, et al. Prognostic assessment of stable coronary artery disease as determined by coronary computed tomography angiography: a Danish multicentre cohort study. Eur Heart J 2017;38:413-21.

35. Hoffmann U, Bamberg F, Chae CU, et al. Coronary Computed Tomography Angiography For Early Triage of Patients with Acute Chest Pain - The Rule Out Myocardial Infarction Using Computed Assisted Tomography (ROMICAT) Trial J Am Coll Cardiol 2009;53:1642-50.

36. Achenbach S, Barkhausen J, Beer M, et al. Consensus recommendations of the German Radiology Society (DRG), the German Cardiac Society (DGK) and the German Society for Pediatric Cardiology (DGPK) on the use of cardiac imaging with computed tomography and magnetic resonance imaging. Rofo 2012;184:345-68.

37. Scappaticci FA, Skillings JR, Holden SN, et al. Arterial thromboembolic events in patients with metastatic carcinoma treated with chemotherapy and bevacizumab. J Natl Cancer Inst 2007;99:1232-9.

38. de Haas EC, Oosting SF, Lefrandt JD, et al. The metabolic syndrome in cancer survivors. Lancet Oncol 2010;11:193-203.

39. Prosnitz RG, Hubbs JL, Evans ES, et al. Prospective assessment of radiotherapy-associated cardiac toxicity in breast cancer patients: analysis of data 3 to 6 years after treatment. Cancer 2007;110:1840-50.

40. Haugnes HS, Wethal T, Aass N, et al. Cardiovascular risk factors and morbidity in long-term survivors of testicular cancer: a 20-year follow-up study. J Clin Oncol 2010;28:4649-57.

41. van Nimwegen FA, Schaapveld M, Cutter DJ, et al. Radiation Dose-Response Relationship for Risk of Coronary Heart Disease in Survivors of Hodgkin Lymphoma. J Clin Oncol 2016;34:235-43.

42. McEniery PT, Dorosti K, Schiavone WA, et al. Clinical and angiographic features of coronary artery disease after chest irradiation. Am J Cardiol 1987;60:1020-4.

43. Roffi M, Patrono C, Collet JP, et al. 2015 ESC Guidelines for the management of acute coronary syndromes in patients presenting without persistent ST-segment elevation: Task Force for the Management of Acute 
Coronary Syndromes in Patients Presenting without Persistent ST-Segment Elevation of the European Society of Cardiology (ESC). Eur Heart J 2016;37:267-315.

44. Detrano R, Guerci AD, Carr JJ, et al. Coronary calcium as a predictor of coronary events in four racial or ethnic groups. N Engl J Med 2008;358:1336-45.

45. Geisel MH, Bauer M, Hennig F, et al. Comparison of coronary artery calcification, carotid intima-media thickness and ankle-brachial index for predicting 10-year incident cardiovascular events in the general population. Eur Heart J 2017;38:1815-22.

46. Yeboah J, McClelland RL, Polonsky TS, et al. Comparison of novel risk markers for improvement in cardiovascular risk assessment in intermediate-risk individuals. Jama 2012;308:788-95.

47. Mahabadi AA, Mohlenkamp S, Lehmann N, et al. CAC Score Improves Coronary and CV Risk Assessment Above Statin Indication by ESC and AHA/ACC Primary Prevention Guidelines. JACC Cardiovasc Imaging 2017;10:143-53.

48. Mahabadi AA, Samy B, Seneviratne SK, et al. Quantitative assessment of left atrial volume by electrocardiographicgated contrast-enhanced multidetector computed tomography. J Cardiovasc Comput Tomogr 2009;3:80-7.

49. Mahabadi AA, Truong QA, Schlett CL, et al. Axial area and anteroposterior diameter as estimates of left atrial size using computed tomography of the chest: comparison with 3-dimensional volume. J Cardiovasc Comput Tomogr 2010;4:49-54.

50. Dykun I, Mahabadi AA, Lehmann N, et al. Left ventricle size quantification using non-contrast-enhanced cardiac computed tomography - association with cardiovascular risk factors and coronary artery calcium score in the general population: The Heinz Nixdorf Recall Study. Acta Radiol 2015;56:933-42.

51. Kalsch H, Lehmann N, Mohlenkamp S, et al. Prevalence of thoracic aortic calcification and its relationship to cardiovascular risk factors and coronary calcification in an unselected population-based cohort: the Heinz Nixdorf Recall Study. Int J Cardiovasc Imaging 2013;29:207-16.

52. Baumgartner H, Falk V, Bax JJ, et al. 2017 ESC/EACTS Guidelines for the management of valvular heart disease. Eur Heart J 2017;38:2739-91.

53. Suh SY, Rha SW, Kim JW, et al. The usefulness of three-dimensional multidetector computed tomography to delineate pericardial calcification in constrictive pericarditis. Int J Cardiol 2006;113:414-6.

54. Hoffmann MH, Shi H, Lieberknecht M, et al. Images in cardiovascular medicine. Sixteen-slice computed tomography and magnetic resonance imaging of calcified pericardium. Circulation 2003;108:e48-9.

55. Rifkin RD, Mernoff DB. Noninvasive evaluation of pericardial effusion composition by computed tomography. Am Heart J 2005;149:1120-7.

56. Barrett CD, Di Biase L, Natale A. How to identify and treat patient with pulmonary vein stenosis post atrial fibrillation ablation. Curr Opin Cardiol 2009;24:42-9.

57. Burgstahler C, Trabold T, Kuettner A, et al. Visualization of pulmonary vein stenosis after radio frequency ablation using multi-slice computed tomography: initial clinical experience in 33 patients. Int J Cardiol 2005;102:287-91.

58. Plana JC, Galderisi M, Barac A, et al. Expert consensus for multimodality imaging evaluation of adult patients during and after cancer therapy: a report from the American Society of Echocardiography and the European Association of Cardiovascular Imaging. J Am Soc Echocardiogr 2014;27:911-39.

59. Vasu S, Hundley WG. Understanding cardiovascular injury after treatment for cancer: an overview of current uses and future directions of cardiovascular magnetic resonance. J Cardiovasc Magn Reson 2013;15:66.

60. Authors/Task Force m, Windecker S, Kolh P, et al. 2014 ESC/EACTS Guidelines on myocardial revascularization: The Task Force on Myocardial Revascularization of the European Society of Cardiology (ESC) and the European Association for Cardio-Thoracic Surgery (EACTS) Developed with the special contribution of the European Association of Percutaneous Cardiovascular Interventions (EAPCI). Eur Heart J 2014;35:2541-619.

61. Kunze KP, Nekolla SG, Rischpler C, et al. Myocardial perfusion quantification using simultaneously acquired ${ }^{13}$ $\mathrm{NH}_{3}$-ammonia PET and dynamic contrast-enhanced MRI in patients at rest and stress. Magn Reson Med 2018. [Epub ahead of print].

62. Biglands JD, Magee DR, Sourbron SP, et al. Comparison of the Diagnostic Performance of Four Quantitative Myocardial Perfusion Estimation Methods Used in Cardiac MR Imaging: CE-MARC Substudy. Radiology 2015;275:393-402.

63. Wentland AL, Grist TM, Wieben O. Review of MRIbased measurements of pulse wave velocity: a biomarker of arterial stiffness. Cardiovasc Diagn Ther 2014;4:193-206.

64. Zhang S, Liu X, Bawa-Khalfe T, et al. Identification of the molecular basis of doxorubicin-induced cardiotoxicity. Nat Med 2012;18:1639-42.

65. Grover S, Lou PW, Bradbrook C, et al. Early and late 
changes in markers of aortic stiffness with breast cancer therapy. Intern Med J 2015;45:140-7.

66. Oberholzer K, Kunz RP, Dittrich M, et al. Anthracyclineinduced cardiotoxicity: cardiac MRI after treatment for childhood cancer. Rofo 2004;176:1245-50.

67. Grover S, Leong DP, Chakrabarty A, et al. Left and right ventricular effects of anthracycline and trastuzumab chemotherapy: a prospective study using novel cardiac imaging and biochemical markers. Int J Cardiol 2013;168:5465-7.

68. Tham EB, Haykowsky MJ, Chow K, et al. Diffuse myocardial fibrosis by T1-mapping in children with subclinical anthracycline cardiotoxicity: relationship to exercise capacity, cumulative dose and remodeling. J Cardiovasc Magn Reson 2013;15:48.

69. Neilan TG, Coelho-Filho OR, Shah RV, et al. Myocardial extracellular volume by cardiac magnetic resonance imaging in patients treated with anthracycline-based chemotherapy. Am J Cardiol 2013;111:717-22.

70. Fallah-Rad N, Walker JR, Wassef A, et al. The utility of cardiac biomarkers, tissue velocity and strain imaging, and cardiac magnetic resonance imaging in predicting early left ventricular dysfunction in patients with human epidermal growth factor receptor II-positive breast cancer treated with adjuvant trastuzumab therapy. J Am Coll Cardiol 2011;57:2263-70.

71. Neilan TG, Coelho-Filho OR, Pena-Herrera D, et al. Left ventricular mass in patients with a cardiomyopathy after treatment with anthracyclines. Am J Cardiol 2012;110:1679-86.

72. Grothues F, Moon JC, Bellenger NG, et al. Interstudy reproducibility of right ventricular volumes, function, and mass with cardiovascular magnetic resonance. Am Heart J 2004;147:218-23.

73. Drafts BC, Twomley KM, D'Agostino R, Jr., et al. Low to moderate dose anthracycline-based chemotherapy is associated with early noninvasive imaging evidence of subclinical cardiovascular disease. JACC Cardiovasc Imaging 2013;6:877-85.

74. Wassmuth R, Lentzsch S, Erdbruegger U, et al. Subclinical cardiotoxic effects of anthracyclines as assessed by magnetic resonance imaging-a pilot study. Am Heart J 2001;141:1007-13.

75. Romano S, Judd RM, Kim RJ, et al. Association of Feature-Tracking Cardiac Magnetic Resonance Imaging Left Ventricular Global Longitudinal Strain With All-Cause Mortality in Patients With Reduced
Left Ventricular Ejection Fraction. Circulation 2017;135:2313-5.

76. Rischpler C, Nekolla SG, Dregely I, et al. Hybrid PET/MR imaging of the heart: potential, initial experiences, and future prospects. J Nucl Med 2013;54:402-15.

77. Imbert L, Marie PY. CZT cameras: A technological jump for myocardial perfusion SPECT. J Nucl Cardiol 2016;23:894-6.

78. Hesse B, Lindhardt TB, Acampa W, et al. EANM/ESC guidelines for radionuclide imaging of cardiac function. Eur J Nucl Med Mol Imaging 2008;35:851-85.

79. Ewer MS, Vooletich MT, Durand JB, et al. Reversibility of trastuzumab-related cardiotoxicity: new insights based on clinical course and response to medical treatment. J Clin Oncol 2005;23:7820-6.

80. DePuey EG, Nichols K, Dobrinsky C. Left ventricular ejection fraction assessed from gated technetium-99msestamibi SPECT. J Nucl Med 1993;34:1871-6.

81. Rischpler C, Totzeck M. Are you stressed? J Nucl Cardiol 2018. [Epub ahead of print].

82. Gayed IW, Liu HH, Yusuf SW, et al. The prevalence of myocardial ischemia after concurrent chemoradiation therapy as detected by gated myocardial perfusion imaging in patients with esophageal cancer. J Nucl Med 2006;47:1756-62.

83. Zellars R, Bravo PE, Tryggestad E, et al. SPECT analysis of cardiac perfusion changes after whole-breast/chest wall radiation therapy with or without active breathing coordinator: results of a randomized phase 3 trial. Int J Radiat Oncol Biol Phys 2014;88:778-85.

84. Gardner SF, Lazarus HM, Bednarczyk EM, et al. Highdose cyclophosphamide-induced myocardial damage during BMT: assessment by positron emission tomography. Bone Marrow Transplant 1993;12:139-44.

85. Zyromska A, Malkowski B, Wisniewski T, et al. (15) $\mathrm{O}-\mathrm{H} 2 \mathrm{O} \mathrm{PET} / \mathrm{CT}$ as a tool for the quantitative assessment of early post-radiotherapy changes of heart perfusion in breast carcinoma patients. Br J Radiol 2018;91:20170653.

86. Song J, Yan R, Wu Z, et al. 13N-Ammonia PET/CT Detection of Myocardial Perfusion Abnormalities in Beagle Dogs After Local Heart Irradiation. J Nucl Med 2017;58:605-10.

87. Bennink RJ, van den Hoff MJ, van Hemert FJ, et al. Annexin $\mathrm{V}$ imaging of acute doxorubicin cardiotoxicity (apoptosis) in rats. J Nucl Med 2004;45:842-8.

88. Panjrath GS, Jain D. Monitoring chemotherapy-induced cardiotoxicity: role of cardiac nuclear imaging. J Nucl 
Cardiol 2006;13:415-26.

89. Panjrath GS, Patel V, Valdiviezo CI, et al. Potentiation of Doxorubicin cardiotoxicity by iron loading in a rodent model. J Am Coll Cardiol 2007;49:2457-64.

90. Estorch M, Carrio I, Berna L, et al. Indium-111antimyosin scintigraphy after doxorubicin therapy in patients with advanced breast cancer. J Nucl Med 1990;31:1965-9.

91. Carrio I, Lopez-Pousa A, Estorch M, et al. Detection of doxorubicin cardiotoxicity in patients with sarcomas by indium-111-antimyosin monoclonal antibody studies. J Nucl Med 1993;34:1503-7.

92. Carrio I, Estorch M, Berna L, et al. Assessment of anthracycline-induced myocardial damage by quantitative indium 111 myosin-specific monoclonal antibody studies. Eur J Nucl Med 1991;18:806-12.

93. Valdes Olmos RA, Carrio I, Hoefnagel CA, et al. High sensitivity of radiolabelled antimyosin scintigraphy in assessing anthracycline related early myocyte damage preceding cardiac dysfunction. Nucl Med Commun 2002;23:871-7.

94. Maini CL, Sciuto R, Ferraironi A, et al. Clinical relevance of radionuclide angiography and antimyosin immunoscintigraphy for risk assessment in epirubicin cardiotoxicity. J Nucl Cardiol 1997;4:502-8.

95. Nousiainen T, Vanninen E, Jantunen E, et al. Anthracycline-induced cardiomyopathy: long-term effects on myocardial cell integrity, cardiac adrenergic innervation and fatty acid uptake. Clin Physiol 2001;21:123-8.

96. Penafiel Ramirez A, Pardo Masferrer J, Pena Viloria C. Unexpected accumulation of $111 \mathrm{In}$-antimyosin in the chest in an area corresponding to the radiation field of a squamous cell lung carcinoma. Rev Esp Med Nucl 1999;18:204-8.

97. Chareonthaitawee P, Beanlands RS, Chen W, et al. Joint SNMMI-ASNC Expert Consensus Document on the Role of (18)F-FDG PET/CT in Cardiac Sarcoid Detection and Therapy Monitoring. J Nucl Med 2017;58:1341-53.

98. Rischpler C, Dirschinger RJ, Nekolla SG, et al. Prospective Evaluation of 18F-Fluorodeoxyglucose Uptake in Postischemic Myocardium by Simultaneous Positron Emission Tomography/Magnetic Resonance Imaging as a Prognostic Marker of Functional Outcome. Circ Cardiovasc Imaging 2016;9:e004316.

99. O'Farrell AC, Evans R, Silvola JM, et al. A Novel Positron Emission Tomography (PET) Approach to Monitor Cardiac Metabolic Pathway Remodeling in Response to
Sunitinib Malate. PLoS One 2017;12:e0169964.

100. Toubert ME, Vercellino L, Faugeron I, et al. Fatal heart failure after a 26-month combination of tyrosine kinase inhibitors in a papillary thyroid cancer. Thyroid 2011;21:451-4.

101. Gorla AK, Sood A, Prakash G, et al. Substantial Increase in Myocardial FDG Uptake on Interim PET/CT May Be an Early Sign of Adriamycin-Induced Cardiotoxicity. Clin Nucl Med 2016;41:462-3.

102. Borde C, Kand P, Basu S. Enhanced myocardial fluorodeoxyglucose uptake following Adriamycinbased therapy: Evidence of early chemotherapeutic cardiotoxicity? World J Radiol 2012;4:220-3.

103. Bauckneht M, Ferrarazzo G, Fiz F, et al. Doxorubicin Effect on Myocardial Metabolism as a Prerequisite for Subsequent Development of Cardiac Toxicity: A Translational (18)F-FDG PET/CT Observation. J Nucl Med 2017;58:1638-45.

104. Bauckneht M, Morbelli S, Fiz F, et al. A Score-Based Approach to (18)F-FDG PET Images as a Tool to Describe Metabolic Predictors of Myocardial Doxorubicin Susceptibility. Diagnostics (Basel) 2017;7.

105. Yan R, Song J, Wu Z, et al. Detection of Myocardial Metabolic Abnormalities by 18F-FDG PET/CT and Corresponding Pathological Changes in Beagles with Local Heart Irradiation. Korean J Radiol 2015;16:919-28.

106. Evans JD, Gomez DR, Chang JY, et al. Cardiac $18 \mathrm{~F}$-fluorodeoxyglucose uptake on positron emission tomography after thoracic stereotactic body radiation therapy. Radiother Oncol 2013;109:82-8.

107.Flotats A, Carrio I, Agostini D, et al. Proposal for standardization of 123I-metaiodobenzylguanidine (MIBG) cardiac sympathetic imaging by the EANM Cardiovascular Committee and the European Council of Nuclear Cardiology. Eur J Nucl Med Mol Imaging 2010;37:1802-12.

108. Wakasugi S, Wada A, Hasegawa Y, et al. Detection of abnormal cardiac adrenergic neuron activity in adriamycin-induced cardiomyopathy with iodine-125metaiodobenzylguanidine. J Nucl Med 1992;33:208-14.

109. Lekakis J, Prassopoulos V, Athanassiadis P, et al. Doxorubicin-induced cardiac neurotoxicity: study with iodine 123-labeled metaiodobenzylguanidine scintigraphy. J Nucl Cardiol 1996;3:37-41.

110. Wakasugi S, Fischman AJ, Babich JW, et al. Metaiodobenzylguanidine: evaluation of its potential as a tracer for monitoring doxorubicin cardiomyopathy. J Nucl Med 1993;34:1283-6. 
111. Boutagy NE, Sinusas AJ. Recent Advances and Clinical Applications of PET Cardiac Autonomic Nervous System Imaging. Curr Cardiol Rep 2017;19:33.

112.Kenk M, Thackeray JT, Thorn SL, et al. Alterations

Cite this article as: Mahabadi AA, Rischpler C. Cardiovascular imaging in cardio-oncology. J Thorac Dis 2018;10(Suppl 35):S4351-S4366. doi: 10.21037/jtd.2018.10.92 of pre- and postsynaptic noradrenergic signaling in a rat model of adriamycin-induced cardiotoxicity. J Nucl Cardiol 2010;17:254-63. 